\title{
Practical Implementation of Green Infrastructure Strategies
}

\author{
John S. Gulliver \\ University of Minnesota, USA \\ 500 Pillsbury Drive S.E., Minneapolis, MN 55455-0116 \\ gulli003@umn.edu
}

\begin{abstract}
Green Infrastructure has many positive attributes, but also some new challenges for a stormwater management system. This seminar will discuss these attributes and challenges and how to overcome the challenges of green infrastructure in stormwater management, including infiltration practices, filtration practices and stormwater ponds.
\end{abstract}

